\title{
Weight change over 14 years in meat-eaters, pescatarians, vegetarians and vegans
}

\author{
A. Knüppel ${ }^{1}$, P. N. Appleby ${ }^{1}$ and T. J. Key ${ }^{1}$ \\ ${ }^{1}$ Cancer Epidemiology Unit, Nuffield Department of Population Health, University of Oxford, OX3 $7 L F$.
}

Meat-free diets have been shown to be associated with lower weight gain short-term ${ }^{(1)}$. The aim of this study was to investigate associations between dietary habits and weight gain over 14 years in the European Prospective Investigation into Cancer and Nutrition (EPIC)-Oxford cohort.

The analysis included data from 10,687 women and 2,516 men <60 years of age at follow-up (20-48 years at baseline). Dietary habits were categorised as meat-eating (3781 women, 903 men), pescatarian (ate fish but not meat; 1109 women, 209 men), vegetarians (2729 women, 711 men) and vegans ( 200 women, 125 men), if these diets were followed both at baseline and follow-up. Participants who were not assigned the same category at baseline and follow-up were categorised as 'reverters' for any step in the direction from vegan to meat-eating (2394 women, 451 men) and 'converters' for any step in the direction from meat-eating to vegan (474 women, 118 men). Weight was self-reported at baseline and follow-up and validated in a sub-sample ${ }^{(2)}$. Mean annual weight gain was multiplied by the mean follow-up time of 14 years. Linear regression was used to compare mean weight gain across diets, adjusting for age, ethnicity, current job, age leaving school, marital status, smoking, physical activity, alcohol intake and body mass index, and stratified by sex.

Mean 14 year weight gain was 4.9 (SD 8.0) $\mathrm{kg}$ in women and 4.9 (SD 7.4) $\mathrm{kg}$ in men. Sustained pescatarians (women $\mathrm{p}<0.001$, men $\mathrm{p}<0.05$ ), vegans (women $\mathrm{p}<0.001$, men $\mathrm{p}<0.05$ ) and those converting towards lower animal product intake (women $\mathrm{p}<0.001$, men $\mathrm{p}$ $<0.01)$ gained significantly less weight than women and men who ate meat (Figure 1). Additionally, vegetarian men gained less weight than meat-eating men $(\mathrm{p}=0.040)$.
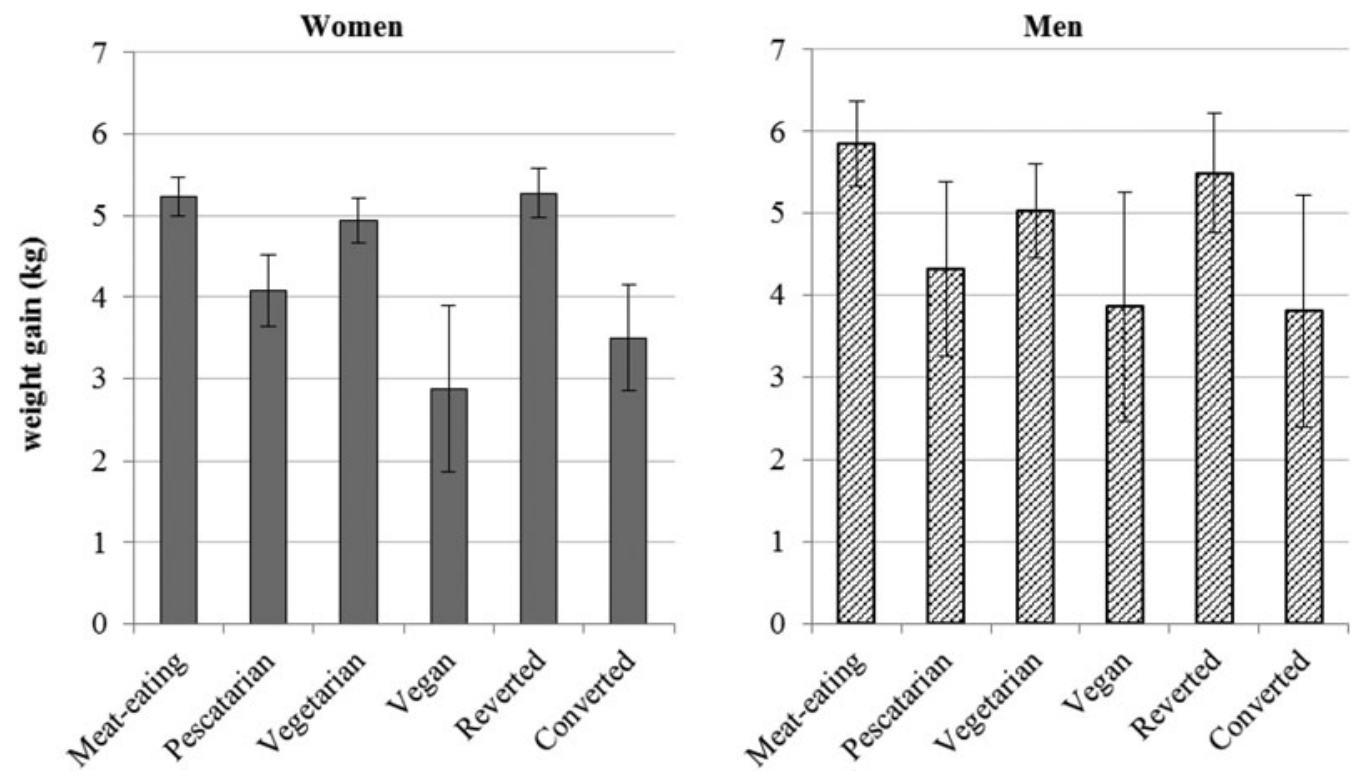

Fig. 1. Mean weight gain $(\mathrm{kg})$ over 14 years by diet group (multivariable adjusted)

The study findings suggest that a reduction in animal products, sustained pescatarian and vegan diets are associated with lower long-term weight gain in adulthood compared with diets that include meat.

This work was supported by the Wellcome Trust Our Planet Our Health (Livestock, Environment and People, LEAP 205212/Z/16/Z).

1. Rosell M, Appleby P, Spencer E et al. (2006) Weight gain over 5 years in 21966 meat-eating, fish-eating, vegetarian, and vegan men and women in EPIC-Oxford. Int J Obes 30, 1389-1396.

2. Spencer EA, Appleby PN, Davey GK et al. (2007) Validity of self-reported height and weight in 4808 EPIC-Oxford participants. Public Health Nutr 5(4), 561-565. 